\title{
Multi-Drug Resistant Tuberculosis Treatment Outcome and Associated Factors in Addis Ababa, Ethiopia: A Retrospective Cross-Sectional Study
}

Tadesse Alemu Bekele ( $\nabla$ talemu55@gmail.com )

Universal Medical and Business College https://orcid.org/0000-0002-4649-7054

Getasew Amogne Aynalem

Wollo University

Trhas Tadesse Berhe

Yekatit 12 Hospital

\section{Research}

Keywords: multi-drug resistant tuberculosis, treatment outcome, factors affecting TB treatment outcome

Posted Date: September 29th, 2020

DOI: https://doi.org/10.21203/rs.3.rs-83241/v1

License: (c) (i) This work is licensed under a Creative Commons Attribution 4.0 International License.

Read Full License 


\section{Abstract}

\section{Background}

Drug-resistant tuberculosis continues to be a public health threat around the globe especially in developing countries. In 2018, the number of new cases of rifampicin-resistant tuberculosis was half a million and about $78 \%$ of them had multi-drug resistant tuberculosis. In Ethiopia, the overall proportion of multi-drug resistant tuberculosis was $11.6 \%$. The latest treatment outcome data for people with multidrug resistant tuberculosis show a global treatment success rate of $56 \%$. Therefore, this study was aimed at assessing multi-drug resistant tuberculosis treatment outcomes and associated factors at St. Peter Specialized Hospital, Ethiopia, 2019.

Methods

A retrospective cross-sectional study was done. A total of 384 patient medical charts selected by simple random sampling method were reviewed. The data was collected using a checklist from the patients' medical charts. The collected data were analyzed with SPSS version 23 computer software package. Summary statistics of a given data for each variable were calculated. A logistic regression model was used to measure the association between the outcome and the predictor variable. Statistical significance was declared at $p$-value $<0.05$. Direction and strength of association were expressed using OR and $95 \% \mathrm{Cl}$.

Result

From a total of 384 patients, the majority of $245(63.8 \%)$ of the study subjects were females. The rate of poor treatment outcome was $173(45.1 \%)$. Male patients and patients in the age category of 39-60 years were less likely to have poor treatment outcome compared to female patients and patients in the age category of $18-38$ years $(A O R=0.43,95 \% \mathrm{Cl}(0.19,0.10)$ and $(\mathrm{AOR}=0.28,95 \% \mathrm{Cl}(0.15,0.52)$ respectively. Patients with a history of second-line TB drug resistance are more likely to have poor treatment outcomes (AOR $=3.81,95 \% \mathrm{Cl}(1.84,7.91)$. Poor treatment outcome was higher among patients with longer treatment duration $(\mathrm{AOR}=3.23,95 \% \mathrm{Cl}(1.82,5.74)$ compared to patients with short treatment duration.

Conclusion

The proportion of poor treatment outcome in patients with multi-drug resistant tuberculosis was high. Therefore, a concerted effort should be done at all levels to improve the treatment outcome of the MDRTB.

\section{Background}

Drug resistance tuberculosis is a category of tuberculosis that is resistant to one or more antituberculosis drugs. It is a fatal communicable disease that poses a serious global health threat. It impacts not only individual patients and their families, but also imposes huge burdens on overextended public health systems that may lack the resources needed to contain it. Multidrug-resistant tuberculosis 
(MDR-TB) refers to TB (tuberculosis) caused by mycobacterium tuberculosis that is resistant to at least two of the most powerful first-line anti-TB medications, isoniazid (INH) and rifampin (RIF) $[1,2]$.

This form of tuberculosis (TB) is considered to be a man-made problem resulting from the consequences of the individual or combined factors related to the management of drug supply, patient management, and patient adherence. It was identified that the occurrence and spread of MDR-TB are mainly associated with behavioral and environmental factors, economic status, and poor infection control practices [3].

In 2018 , the number of new cases of rifampicin-resistant tuberculosis was half a million and about $78 \%$ of them had multi-drug resistant tuberculosis around the globe. The rate of multidrug-resistant TB or rifampicin-resistant was $3.4 \%$ among new TB cases and $18 \%$ among previously treated TB cases worldwide in 2018. In the same year, there were about 214000 (range, 133 000-295 000) deaths from MDR/RR-TB [4].

Ethiopia is one of the 27 countries with a high MDR-TB burden and ranked $15^{\text {th }}$ with new cases of MDRTB each year. The overall proportion of multidrug-resistant tuberculosis (MDR-TB) in the country was $11.6 \%$ (4.3\% in new patients and $6.7 \%$ in previously treated patients). A systematic review of the prevalence of MDR-TB found that the mean rate of MDR-TB in Ethiopia was $12.6 \pm 15.9 \%[5,6,7]$.

A World Health Organization (WHO) report indicated the latest treatment outcome data for people with MDR/RR-TB show a global treatment success rate of $56 \%$. Ethiopia is one of the high MDR-TB burden countries with better treatment success rates $(>70 \%)$. A study done in Ethiopia showed that the overall rate of unfavorable treatment outcomes at the end of the treatment was $21.21 \%[4,8]$.

Even though the cases of MDR-TB are increasing in the health care facilities including the study area, information about MDR-TB treatment outcome and its associated factors is not sufficient. The causes of poor MDR-TB treatment outcomes in different socioeconomic contexts are complex and multi-factorial. Therefore, the study was aimed to assess MDR-TB treatment outcome and associated factors at St. Peter Specialized Hospital, Ethiopia. The finding of this study will help health workers and responsible bodies working on the management of MDR-TB to strengthen the existing uniform treatment guideline or protocol and for planning effective treatment strategies.

\section{Methods}

Study design and setting: A retrospective cross-sectional patient medical chart review was done to assess the treatment outcome of multi-drug resistant tuberculosis and associated factors. The study was done at St. Peter Specialized Hospital. It was established in 1953 E.C and it is the only TB specialized hospital found in Ethiopia. The hospital envisions being one of the centers of excellence and model TB general specialized hospital in East Africa. The data was gathered from June to July 2019.

Sample size and sampling method: The sample size for the study was calculated using a single proportion formula by assuming the proportion of poor treatment outcome to be $50 \%, 5 \%$ marginal error 
with 95\% Cl. Hence, a total of 384 MDR-TB patients medical chart registered from June 2014 to May 2019 were included in this study. A simple random sampling technique was used to select the patient's medical chart. Patients' medical chart with incomplete information was excluded from the study.

Study variables: the dependent variable was the treatment outcome of MDR-TB. The independent variables include demographic factors (age, weight, sex) and clinical characteristics (site of TB infection, TB treatment history, HIV status, TB category, co-morbidities, TB treatment resistance (drugs), smear test result).

Data collection tool and procedure: The data was collected by using a data abstraction checklist. Data was collected from complete medical charts only. Relevant information like demographic characteristics, clinical characteristics, and treatment outcome were collected by reviewing patient medical charts. The data was gathered by two data collectors who were trained for one day before data collection.

Data analysis procedure: The collected data were checked for completeness, coded, entered, and analyzed with Statistical Package for the Social Sciences (SPSS) version 23 computer software package. Summary statistics of a given data for each variable were calculated. A logistic regression model was used to measure the association between the outcome and the predictor variable. Statistical significance was declared at p-value $<0.05$. Direction and strength of association were expressed using OR and $95 \% \mathrm{Cl}$.

\section{Operational definitions:}

\section{Definitions of treatment outcome of Drug-resistant/MDR-TB according to the National Guideline for TB, Drug-Resistant TB, and Leprosy in Ethiopia (9):}

Cured: Treatment completed according to national recommendation without evidence of failure and three or more consecutive cultures taken at least 30 days apart are negative after the intensive phase.

Treatment completed: Treatment completed according to national recommendation without evidence of failure but no record that three or more consecutive cultures taken at least 30 days apart are negative after the intensive phase.

Treatment failure: Treatment terminated or need for permanent regimen change of at least two anti-TB drugs

Died: A patient who dies during the course of TB treatment.

Lost to follow up (LTFU): A patient whose treatment was interrupted for two consecutive months or more. Not Evaluated: A TB patient for whom no treatment outcome is assigned. This includes "transferred out" cases with the unknown outcome at the reporting unit.

Moved: A RR-/MDR-TB patient registered to second-line TB treatment and whose current regimen is now decided to be switched to a newly re-constructed DR-TB regimen before the end of the intensive phase. 


\section{Results}

\section{Socio-demographic Characteristics of the Patients:}

A total of 384 MDR-TB patient medical charts was reviewed and included in the study. The majority, $245(63.8 \%)$ of the study subjects were females and $139(36.2 \%)$ were males. More than three-forth, $306(79.7 \%)$ of the patients were in the age group of 39-60 years with a mean \pm SD of $29.40 \pm 9.81 \mathrm{yrs}$. The weight of most $202(52.6 \%)$ of the patients was in the range of $18-48 \mathrm{Kg}$ with a mean \pm SD of $50.47 \pm 8.02 \mathrm{Kg}$ (table 1).

Table 1: Socio-demographic Characteristics of the Patients, St. Peter Specialized Hospital, Addis Ababa, Ethiopia, 2019

\begin{tabular}{|llll|}
\hline Variable & Category & Frequency & Percent \\
\hline Sex & Male & 139 & 36.2 \\
\cline { 2 - 4 } & Female & 245 & 63.8 \\
\hline Age (Yrs) & $18-38$ & 78 & 20.3 \\
\cline { 2 - 4 } & $39-60$ & 306 & 79.7 \\
\cline { 2 - 4 } & Mean + SD & $29.40 \pm 9.81$ \\
\hline Body weight (Kg) & $38-55$ & 202 & 52.6 \\
\cline { 2 - 4 } & $56-73$ & 182 & 47.4 \\
\cline { 2 - 4 } & Mean $\pm S D$ & $50.47 \pm 8.02$ & \\
\hline
\end{tabular}

\section{Clinical Characteristics of the Patients:}

From a total of 384 patient medical charts reviewed, the majority $340(88.5 \%)$ had pulmonary TB and only a small proportion of $44(11.5 \%)$ of them have had an extrapulmonary TB. About three fourth $292(76.0 \%)$ of them were new cases and $92(23.0 \%)$ retreatment MDR-TB cases. It was found that $288(75.0 \%)$ of the patients were resistant to first-line drugs. Regarding the treatment duration, $110(28.6 \%)$ of the patients were treated for $6-18$ months with a mean \pm SD of $14.52+5.13$ months. More than half $230(59.9 \%)$ of them have HIV/AIDS (Table 2).

Table 2: Clinical Characteristics of the Patients, St. Peter Specialized Hospital, Addis Ababa, Ethiopia, 2019 


\begin{tabular}{|llll|}
\hline Variable & Category & Frequency & Percent \\
\hline Site of TB infection & Pulmonary TB & 340 & 88.5 \\
\cline { 2 - 4 } & Extraplumonary TB & 44 & 11.5 \\
\hline TB treatment history & Retreatment & 292 & 76.0 \\
\cline { 2 - 4 } & New & 92 & 23.0 \\
\hline TB treatment-resistant & First-line & 288 & 75.0 \\
\cline { 2 - 4 } & Second-line & 96 & 25.0 \\
\hline TB treatment Duration & $6-18$ & 110 & 28.6 \\
\cline { 2 - 4 } & $19-30$ & 276 & 71.4 \\
\cline { 2 - 4 } & Mean \pm SD & $14.52+5.13$ & \\
\hline Smear test & Positive & 243 & 63.3 \\
\cline { 2 - 4 } & Negative & 141 & 36.7 \\
\hline HIV Status & Yes & 230 & 59.9 \\
\cline { 2 - 4 } & No & 154 & 40.1 \\
\cline { 2 - 4 } & &
\end{tabular}

\section{Treatment Outcome of MDR-TB:}

The overall treatment outcome of MDR-TB was classified as a good (favorable) outcome if the patient is cured and a poor (unfavorable) outcome if the patient is default or died. From the total 384 MDR-TB patient medical charts reviewed, more than half 211(54.9\%) of them had good treatment outcomes. The rate of unfavorable treatment outcome was found to be high, 173(45.1\%); from which 106(27.6\%) have died of the disease, and $67(17.5 \%)$ have defaulted from the treatment (figure 1 ).

\section{Factors associated with MDR-TB treatment outcome:}

A bivariate and multivariate logistic regression model was carried out to determine the factors affecting the MDR-TB treatment outcome. Only variables with a $p$-value $\leq 0.2$ were included in the multivariate regression. Hence, Age, sex, treatment resistance, duration of treatment, smear result, and HIV status were found to be significantly associated with MDR-TB treatment outcome.

Male patients and patients in the age group of 39-60 years were less likely to have poor outcome compared to female patients and patients in the age category of $18-38$ years $(\mathrm{AOR}=0.43,95 \% \mathrm{Cl}(0.19$, $0.10)$ and $(A O R=0.28,95 \% \mathrm{Cl}(0.15,0.52)$ respectively. Patients who have a history of second-line TB drug resistance are approximately four times to have poor treatment outcomes compared to patients who have a history of first-line TB drugs $(A O R=3.81,95 \% \mathrm{Cl}(1.84,7.91)$. Poor treatment outcome was three times higher among patients with longer duration of treatment (19-30months) ( $A O R=3.23,95 \% \mathrm{Cl}(1.82$, 
5.74) compared to patients with shorter duration of treatment (6-18 months). Patients with a negative smear test results and HIV positive patients were more likely to have poor treatment outcomes compared to patients with a smear-positive test result and HIV negative patients $(A O R=2.57,95 \% \mathrm{Cl}(1.56,4.22)$ and $($ AOR $=1.93,95 \% \mathrm{Cl}(1.15,3.23)($ Table 3$)$.

Table 3: Factors associated with MDR-TB treatment outcomes St. Peter Specialized Hospital, Addis Ababa, Ethiopia, 2019.

\begin{tabular}{|c|c|c|c|c|c|c|}
\hline \multirow[t]{3}{*}{ Characteristics } & & \multicolumn{2}{|c|}{ Treatment Outcome } & \multirow{3}{*}{$\begin{array}{l}\text { COR }(95 \% \\
\text { Cl) }\end{array}$} & \multirow{3}{*}{$\begin{array}{l}\text { AOR( } 95 \% \\
\mathrm{Cl})\end{array}$} & \multirow{3}{*}{$\begin{array}{l}\text { P- } \\
\text { Value }\end{array}$} \\
\hline & & Poor & Good & & & \\
\hline & & (No, \%) & (No, \%) & & & \\
\hline \multirow[t]{2}{*}{ Age (in years) } & $18-38$ & $43(24.9)$ & $35(16.6)$ & 1 & 1 & \multirow[t]{2}{*}{$0.039 *$} \\
\hline & $39-60$ & $130(75.1)$ & $176(83.4)$ & $\begin{array}{l}\text { 1.66(1.01, } \\
2.74)\end{array}$ & $\begin{array}{l}0.43(0.19 \\
0.10)\end{array}$ & \\
\hline \multirow[t]{2}{*}{ Sex } & Male & $86(49.7)$ & $53(25.1)$ & $\begin{array}{l}0.34(0.22 \\
0.52)\end{array}$ & $\begin{array}{l}0.28(0.15 \\
0.52)\end{array}$ & \multirow[t]{2}{*}{0.000 * } \\
\hline & Female & $87(50.3)$ & 158(74.9) & 1 & 1 & \\
\hline \multirow[t]{2}{*}{ Weight (in Kg) } & $38-55$ & $92(53.2)$ & $110(52.1)$ & $\begin{array}{l}0.96(0.64 \\
1.43)\end{array}$ & $\begin{array}{l}1.04(0.64 \\
1.69)\end{array}$ & \multirow[t]{2}{*}{0.880} \\
\hline & $56-73$ & $81(46.8)$ & $101(47.9)$ & 1 & 1 & \\
\hline \multirow{2}{*}{$\begin{array}{l}\text { TB treatment } \\
\text { history }\end{array}$} & New & $148(85.5)$ & $144(68.2)$ & 1 & 1 & \multirow[t]{2}{*}{0.205} \\
\hline & Retreatment & $25(14.5)$ & $67(31.8)$ & $\begin{array}{l}2.75(1.65, \\
4.60)\end{array}$ & $\begin{array}{l}1.53(0.79 \\
2.94)\end{array}$ & \\
\hline \multirow{2}{*}{$\begin{array}{l}\text { Treatment } \\
\text { resistance }\end{array}$} & First line & $143(82.7)$ & $145(68.7)$ & 1 & 1 & \\
\hline & Second line & $30(17.3)$ & $66(31.3)$ & $\begin{array}{l}2.17(1.33 \\
3.54)\end{array}$ & $\begin{array}{l}3.81((1.84, \\
7.91)\end{array}$ & 0.000 * \\
\hline \multirow{2}{*}{$\begin{array}{l}\text { Treatment } \\
\text { duration }\end{array}$} & 6-18months & $62(35.8)$ & $48(22.7)$ & 1 & 1 & \multirow[t]{2}{*}{$0.000 *$} \\
\hline & $\begin{array}{l}19- \\
30 \text { months }\end{array}$ & $111(64.2)$ & 163(77.3) & $\begin{array}{l}\text { 1.90(1.21, } \\
2.97)\end{array}$ & $\begin{array}{l}3.23(1.82 \\
5.74)\end{array}$ & \\
\hline \multirow[t]{2}{*}{ Smear test result } & Positive & $120(69.4)$ & $123(58.3)$ & 1 & 1 & \multirow[t]{2}{*}{$0.000 *$} \\
\hline & Negative & $53(30.6)$ & $88(41.7)$ & $\begin{array}{l}1.62(1.06, \\
2.47)\end{array}$ & $\begin{array}{l}2.57(1.56, \\
4.22)\end{array}$ & \\
\hline \multirow[t]{2}{*}{ HIV Status } & Positive & $87(50.3)$ & $143(67.8)$ & $\begin{array}{l}\text { 2.08(1.37, } \\
3.15)\end{array}$ & $\begin{array}{l}\text { 1.93(1.15, } \\
3.23)\end{array}$ & \multirow[t]{2}{*}{$0.013^{*}$} \\
\hline & Negative & $86(49.7)$ & $68(32.2)$ & 1 & 1 & \\
\hline
\end{tabular}




\section{Discussion}

This study was intended to assess MDR-TB treatment outcomes and predictors of unfavorable treatment outcome among patients who were enrolled in St. Peter Specialized Hospital, Addis Ababa, Ethiopia.

The finding of this study indicated more than half of them (54.9\%) had a good treatment outcome. The rate of poor treatment outcome in this study was $45.1 \%$ which is higher than the study done in China and Turkey but in agreement with the study done in Pakistan and lower than the study that was done in South Africa $[10,11,12,13]$. The inconsistencies of these findings may be related to differences in patient monitoring mechanism, the difference in the referral system, awareness of the patients about the disease condition, and its treatment and difference in the structure of the health system.

In this study, it was found that male patients were less likely to have poor treatment outcomes than female patients. This finding contradicts the study done in South Africa and India in which male patients were predictors of the poor treatment outcome $[11,14]$. This may be due to differences in socio-cultural influences. In Ethiopia, most females are economically dependent on males and have less decision making role than males. This may in turn affect their health, health-seeking behavior, and self-care activities which may lead to poor treatment outcomes in general.

A study done in Turkey showed that young age, HIV status, and no drug resistance were factors significantly associated with successful treatment outcomes. Similarly in this study presence of positive HIV conditions and a history of resistance to second-line drugs were significantly associated with poor treatment outcomes. In contrast to a study done in Turkey and China [12,13], in this study younger age patients were more likely to have poor treatment outcomes. This difference may be due to a smaller proportion of young age patients in this study.

Patients who have a history of second-line anti-TB drug resistance were more likely to have poor treatment outcomes. The finding of this study is in line with studies done in India, Pakistan, and Tanzania $[14,15,16]$.

Poor treatment outcome was three times higher among patients with longer duration of treatment (1930 months). The finding of this study is also supported by a study done in India the odds of poor treatment outcome was higher among patients with longer duration of treatment [14].

Patients with smear test result negative and patients with co-morbid conditions were more likely to have poor treatment outcomes. This finding is in line with studies done in Ethiopia, India, and Pakistan, where patients with smear-negative results have a higher risk of poor treatment outcomes $[8,14,15]$.

\section{Limitation}

This was a cross-sectional study and assessed cause and effect simultaneously. Hence, causal links may not be established due to the lack of temporal connection. This study was also based on the review of records; hence inherent design limitations such as the incompleteness of data were noted. 


\section{Conclusion}

Although the majority of the patients with multi-drug resistant tuberculosis have good treatment outcomes still a high proportion (45.0\%) of them encountered poor treatment outcomes. Age, sex, treatment resistance, duration of treatment, smear result, and co-morbid condition were found to be significantly associated with MDR-TB treatment outcome. Therefore, decisive measures are urgently needed by all concerned bodies to effectively treat MDR-TB cases and improve treatment success and survival. Age, sex, treatment resistance, smear result, duration of treatment, and co-morbid condition are found to be associated with treatment outcome. Hence, providing additional educational and medical support, and closely monitoring the clinical characteristics of these patients by the health professionals can contribute well to treatment success.

\section{Abbreviations}

AOR: Adjusted Odds Ratio

Cl: Confidence Interval

HIV: Human Immunodeficiency Virus

IRB: Institutional Review Board

INH: Isoniazid

LTFU: Lost to follow up

MDR-TB: Multi Drug Resistant Tuberculosis

OR: Odds Ratio

RIF: Rifampin

SPSS: Statistical Package for the Social Sciences

TB: Tuberculosis

UMBC: Universal Medical and Business College

WHO: World Health Organization

\section{Declarations}

\section{Ethics approval and consent to participants:}


Approval and ethical clearance was obtained from the Institution Review Board (IRB) of Universal Medical and Business College (UMBC). Official permission was also obtained from the principals of St. Peter Specialized Hospital before data collection. The objective and purpose of the study was clearly explained to the authorities of the hospital before data collection. Confidentiality of the information was maintained and the data was recorded anonymously throughout the study.

\section{Consent for Publication:}

All authors consent for publication.

\section{Accessibility of data and materials:}

The result of this research was extracted from the data gathered and analyzed based on the stated methods and materials. The datasets used and/or analyzed during the current study are available from the corresponding author on reasonable request.

\section{Conflict of interests:}

The author asserts that there is no conflict of interest regarding the publication of this manuscript.

\section{Funding:}

This research was funded by Universal Medical College but has no other role in the manuscript.

\section{Authors' contributions:}

All authors contributed to data analysis, drafting or revising the article, gave final approval of the version to be published, and agree to be accountable for all aspects of the work.

\section{Acknowledgments:}

Our heartfelt thanks go to Universal Medical and Business College funding the study and St. Peter Specialized Hospital for permission to conduct the study. The researchers also wish to express their gratitude to the study subjects and to all those who lent their hands for the successful completion of this research.

\section{References}

1. Edmund G.Brown, Diana S. Dooley, Karen Smith. Curry International Tuberculosis Center and California Department of Public Health, 2016: Drug-Resistant Tuberculosis: A Survival Guide for Clinicians, $3^{\text {rd }}$

2. Shah NS, Wright A, Bai GH, et al. Worldwide emergence of extensively drug-resistant tuberculosis. Emerg Infect Dis. 2007;13(3):380- 
3. Muluken Desalegn et al. Predictors of multidrug-resistant tuberculosis among adult patients at Saint Peter Specialized Hospital, Addis Ababa, Ethiopia. Pan African Medical Journal. 2016;25(2):5. [doi:10.11604/pamj.supp.2016.25.2.9203].

4. World Health Organization, "WHO report 2019", Global Tuberculosis Control, WHO, Geneva, Switzerland, 2019.

5. Lomøy J \& Dybul m Drug-resistant Tuberculosis. A Growing Global Health Threat. March 29, 2017.

6. Diriba, G, Kebede, A, Tola, $\mathrm{HH}$ et al.Surveillance of drug resistance tuberculosis based on reference laboratory data in Ethiopia. Infect Dis Poverty 8, 54 (2019). https://doi.org/10.1186/s40249-0190554-4

7. Weldegebreal S, Teweldemedhin M, Gebreyesus H. Prevalence of Multidrug-Resistant Tuberculosis and Associated Factors in Ethiopia: A Systematic Review. Hindawi Journal of Pathogens: 2018

8. Woldeyohannes D, Assefa T, Aman R, Tekalegn Y, Hailemariam Z. Predictors of time to unfavorable treatment outcomes among patients with multidrug-resistant tuberculosis in Oromia region, Ethiopia. October. PLoS ONE 2019.14(10). org/10.1371/journal.pone.0224025

9. Federal Ministry of Health (FMOH) of Ethiopia. National Guideline for the Management of TB, Drug Resistant TB and Leprosy. $6^{\text {th }}$ Addis Ababa: FMOH; 2018.

10. Kanwal S, Akhtar AM, Ahmed A. Factors associated with mortality to drug-resistant tuberculosis and their programmatic management in treatment centers of Punjab, Pakistan. Journal of Pakistan Medical Association. 2017, 67(6).

11. Elliott $E$, Draper HR, Baitsiwe $P$, Claassens MM. Factors affecting treatment outcomes in drugresistant tuberculosis cases in the Northern Cape, South Africa. Public Health Action. 2014;4(3):201DOI:10.5588/pha. 14.0029

12. Sengul A, Akturk UA, Aydemir Y, Kaya N, Kocak ND, Tasolar FT (2015). Factors affecting successful treatment outcome in pulmonary tuberculosis: a single-center experience in Turkey, 2005-2011. J Infect Dev Ctries 9:821-828. DIO: 10.3855/jidc.5925.

13. Zhang L, Meng Q, Chen S, et al. Treatment outcomes of multidrug-resistant tuberculosis patients in Zhejiang, China, 2009-2013. Clin Microbiol Infect. 2018;24(4):381- DOI:10.1016/j.cmi.2017.07.008

14. Parmar MM, Sachdeva KS, Dewan PK, et al. Unacceptable treatment outcomes and associated factors among India's initial cohorts of multidrug-resistant tuberculosis (MDR-TB) patients under the revised national TB control program (2007-2011): Evidence leading to policy enhancement. PLoS One. 2018;13(4):e0193903. Published 2018 Apr 11. DOl:10.1371/journal.pone.0193903

15. Javaid A, Ullah I, Masud H, et al. Predictors of poor treatment outcomes in multidrug-resistant tuberculosis patients: a retrospective cohort study. Clin Microbiol Infect. 2018;24(6):612DOI:10.1016/j.cmi.2017.09.012

16. Leveri TH, Lekule I, Mollel E, Lyamuya F, and Kilonzo K. Predictors of Treatment Outcomes among Multidrug ResistantTuberculosis Patients in Tanzania.Hindawi. Tuberculosis Research and Treatment. Volume 2019, Article ID 3569018, 10 pages.https://doi.org/10.1155/2019/3569018 
Figures

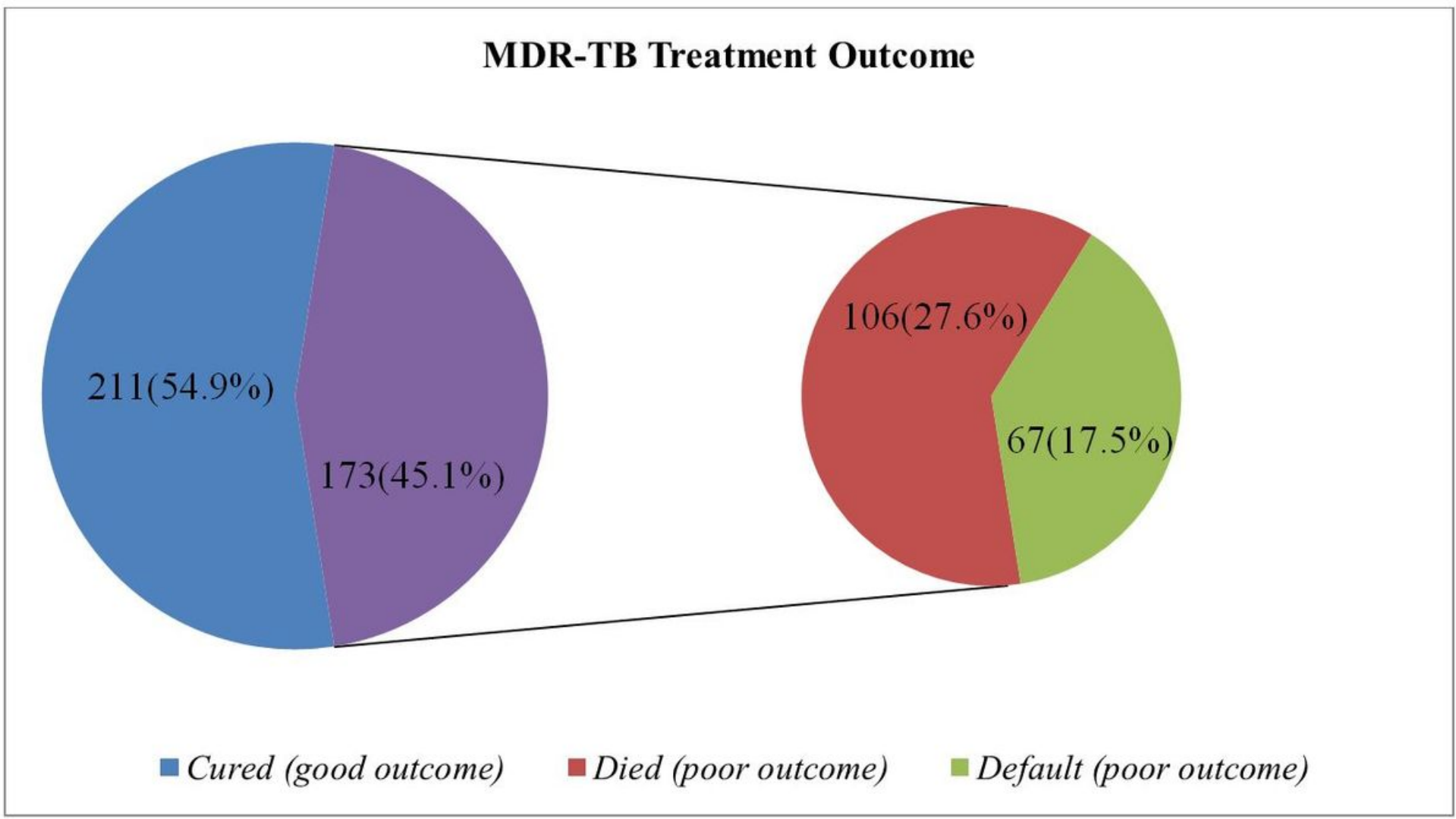

\section{Figure 1}

Treatment outcome of multidrug-resistant tuberculosis (MDR-TB), St. Peter Specialized Hospital, Addis Ababa, Ethiopia, 2019. 